\title{
Modeling and Forecasting Ocean Acoustic Conditions
}

\author{
by Timothy F. Duda ${ }^{1,2}$
}

\begin{abstract}
Modeling acoustic conditions in an oceanic environment is a multiple-step process. The environmental conditions (features) in the area first must be measured or estimated; relevant features include seabed geometry, seabed composition, and four-dimensionally (4D) variable sound-speed and density variations related to evolving or wave motions. Often the dynamical wave modeling depends on first obtaining correct seabed and mean stratification conditions (for example, nonlinear internal wave modeling). Next, this information must be included in sound propagation modeling. A selection of the many methods and tools available for these tasks are described, with a focus on modeling sounds of 20 to $1000 \mathrm{~Hz}$ propagating through water-column features that are time-dependent and variable in three dimensions (i.e., 4D variable). An example of a 3D parabolic equation acoustic calculation shows how variability caused by evolving internal tidal waves affects sound propagation. Different propagation and scattering regimes are discussed, including the theoretically delineated weak scattering and strong scattering regimes, as well as the empirically examined regime found in nonlinear internal waves. The histories and the current state of our oceanographic knowledge (the input to acoustic modeling) and of our ability to effectively model complex acoustic conditions are discussed. Example acoustic simulation applications are also discussed; these are ocean acoustic tomography, coherence prediction, and signal-to-noise ratio prediction. Types of ocean models and acoustic models and how they are interfaced are also examined. These include deterministic, statistical analytic feature models.

Keywords: Acoustic coherence, acoustic statistics, dynamical ocean modeling, internal tides, nonlinear internal waves, ocean acoustic modeling, parabolic equation, seabed interacting sound.
\end{abstract}

\section{Introduction}

Practical application of data-constrained, geophysical dynamical models is straightforward: for processes occurring in a volume, the best knowledge of conditions within the volume will yield the best process forecast. Underwater acoustics is one such process that is of major interest to many technical communities, Underwater acoustics is a primary naval technology; marine mammals use sound, are impacted by changes to the sonic environment, and can be located and studied via their vocalizations. Industrial marine technologies

1. Woods Hole Oceanographic Institution, Applied Ocean Physics and Engineering Department, Woods Hole, Massachusetts 02543, USA

2. Corresponding author: e-mail: tduda@whoi.edu 
such as transportation, fossil fuel extraction, mining, and power generation emit sound whose impacts can be better understood by model-based forecasting. Ocean processes can be studied with sound, passively in the case of measurement of sounds from storms, rain, earthquakes, and polar ice features, and actively in ocean acoustic tomography and thermometry. Underwater acoustic forecasting and prediction in support of these activities and basic research are obvious candidates for application of ocean dynamical models of types covered in this book. This is because time-varying oceanic thermal and salinity gradients can have fundamental effects on sound (Robinson and Lee 1994; Brekhovskikh and Lysanov 2001). Such acoustic modeling shares a need for accurate dynamical forecasts with other applications, including prediction of harmful algal blooms (Anderson et al. 2012), reverse integration of oceanic drift paths of aircraft debris to estimate a crash site, oil spill dispersal modeling, and use of modeled atmospheric or ocean flow models to optimally route aircraft or ships. Each of these dynamical model predictive applications places specific constraints on what constitutes an effective dynamical model. The objectives can be met when accurate and inclusive models are used, but employing inaccurate models or models with inadequate resolution may be fruitless or even detrimental.

The concept behind linking environmental models and acoustics is simple: for effective research or skillful operational forecasting, use the best possible models to compute acoustic fields given environmental information, and use the best models to provide that information. However, the simplicity of the concept belies the challenging nature of the work. This review cannot address completely the complexities of ocean acoustic modeling and prediction. Instead, the major underlying principles are described, with illustrative examples and a few applications.

Here, basic concepts regarding transmission of sound at 20 to $1000 \mathrm{~Hz}$ through the ocean are presented. Sound at these frequencies is not strongly absorbed by seawater. Further, it shows complicated long-range and/or bottom-interacting propagation behavior that differs from the simpler behavior of some familiar acoustic applications, such as echo sounders for bathymetric measurement or water-column scattering studies, acoustic Doppler current profilers, and inverted echo sounders. Those familiar applications are somewhat insensitive to some detail of the propagation medium. They typically treat sound propagation paths as straight (or nearly so), and mainly benefit from knowledge of local sound-speed conditions for accurate conversion of wave travel time to distance.

In the following sections, the four-dimensionally (4D) variable ocean environment is discussed and a computational acoustic example presented. The intuitive idea that better ocean knowledge allows for better understanding of acoustic conditions is then explored. Some specific acoustic behaviors in ocean features are then examined for the purpose of illustrating which ocean factors must be correctly known (modeled). Various methods used to capture this knowledge for acoustic modeling are examined. These include both deterministic and stochastic modeling of ocean features and acoustic properties. (Even stochastic models of acoustic properties, such as phase variance, which can optionally use stochastic ocean models, need quality information about the background within which 
the stochastic ocean processes are embedded.) Potential future improvements to coupled dynamical and acoustic models that would allow that pairing to better capture the essence of time-evolving acoustic behavior are also briefly mentioned throughout the text.

\section{Simulating the four-dimensional underwater sound propagation environment}

\section{a. Equations and approximations}

The sound field at a place and time is described by pressure and velocity of traveling or evanescent waves. For narrowband sound, the field to be modeled is described by pressure amplitude and phase, which are both of interest. For example, adequate amplitude and stability (temporal coherence) of phase are key to source detection and tracking. The spatial coherences of these are also of interest because they may affect array signal processing performance. For wider band systems, such as for acoustic communication or tomography, waveform (impulse response) predictions-for example, for providing multipath stability predictions - can supply key information.

Undersea sound propagation is somewhat paradoxical. One linear governing pressurewave equation describes the physical behavior in the water column with a high degree of accuracy while, at the same time, the relevant conditions in an ocean volume of acoustic interest can have unlimited complexity. The apparent simplicity suggested by the single equation is only superficial, owing to the intricacy and uncertainty of two things: the geometrical water domain in which the equation is applied, and the spatiotemporal structure of the in-water sound-speed coefficient. The wave equation stems from the equation for sound pressure $(p)$ in a medium with current $(\mathbf{U})$ and density $\left(\rho_{0}\right)$,

$$
(\partial / \partial t+\mathbf{U} \cdot \nabla)^{2} p=c^{2} \rho_{0} \nabla\left(\rho_{0}{ }^{-1} \nabla p\right)
$$

(Colosi, 2016). This calculation reduces to the familiar governing wave equation if the current and the density gradient are each set to zero,

$$
\frac{\partial^{2} p(x, y, z, t)}{\partial t^{2}}=c^{2}(x, y, z, t) \nabla^{2} p(x, y, z, t)
$$

Density gradients contribute greatly to backscatter but are typically ignored in propagation studies. The effect of current on sound propagation has been analyzed in few studies typically involving sound travel time from point to point, but is often not considered. The multidimensional structure of the sound speed $c(x, y, z, t)$ creates solutions of notable complexity for even a single known source of sound, let alone for a collection of sources, with either known or partially known properties. The wave equation reduces to the Helmholtz equation for a harmonic signal of angular frequency $\omega$, i.e., for the case $p=p_{0} \exp (-i \omega t)$. In many instances, three-dimensional (3D) solutions are necessary due to seafloor interaction (Finette et al. 2007; Lin et al. 2015) or to strong lateral gradients of $c$ (Finette et al 2007; Lynch et al. 2010; Duda et al. 2011a, b) so that the modeling of sound from individual sources and 
the modeling of noise (meant to characterize sound from numerous, imprecisely localized sources), can be computationally intensive and subject to large uncertainties.

The sound speed in water is generally treated with a high degree of accuracy as a realvalued quantity that is independent of $\omega$, but depends in a known manner on temperature, salinity and pressure (Del Grosso 1974; Dushaw et al. 1993). (Effects from temperature fluctuations usually dominate over those of salinity fluctuations, except of course in unique, near-isothermal salt-stratified regions such as at high latitude.) With this treatment, attenuation of high-frequency sound that is strongly absorbed in seawater can be handled with a simple method: multiplication by an along-path, integrated, frequency-dependent absorption rate that also depends on temperature, salinity, $\mathrm{pH}$, and pressure (Francois and Garrison 1982; Brewer et al. 1995). Absorption also can be treated by generalizing $c$ to be a complex number, equivalent to a complex wavenumber $k=\omega / c$, where the fractional absorption per unit of meter distance is governed by the imaginary component of the wavenumber in a straightforward manner.

The frozen-field approximation is used for most ocean acoustic modeling. A notable exception is modeling of sound reflecting from the moving ocean surface, a process that leads to detectable Doppler shifting (Deane et al. 2012), although examples of this implementation are uncommon in the literature. I refer here to time-stepped, frozen-field modeling of underwater sound as 3.5D simulation. Because sound moves at about $1.5 \mathrm{~km} / \mathrm{s}$ in the ocean, pulsed sound traveling hundreds of kilometers could, in principle, be modeled by updating the medium conditions over the excursion time of hundreds of seconds as the sound moves away from the source. But most dynamical models covering hundreds of kilometers are not intended to faithfully simulate the details of processes that alter the ocean much over a few hundred seconds. Thus, modeling sound in a moving ocean with current-era models is not compelling.

Further complexity arises from the nature of the seabed. Sound energy in the water (where it is most commonly sensed in oceanic or estuarine applications, and emitted in active systems) can strongly interact with the seabed by reflecting from it or by penetrating into it and then radiating back into the water. The ramifications, in the water column, of the effective reflection and penetration and of surface and bubble-cloud interactions, are frequency-dependent and are the subject of many decades of research, too detailed to cover here. The elastic or poro-elastic nature of the seabed is often disregarded in ocean sound propagation models, and a dense fluid intended to mimic the true medium is often substituted, allowing use of the wave (or Helmholtz) equation. This substitution can be inadequate to model some significant effects in some situations, and is remedied by solving a more complete set of equations for the sound-related motions in the coupled water-seabed system (Yamamoto 1983; Collins 1991; Collins et al. 1995, 1997; Collis et al. 2008). The fluid seabed model is adopted here for discussions and illustrations that emphasize the variability and time-evolution of the aqueous ocean. The fluid seabed model is fairly conventional (see, for example, Yevick and Thomson 1997, who discuss the treatment of the density discontinuity at the interface of water and [denser] fluid seabed). The methods for 
modeling the complete physics, which allow waves enabled by a non-zero shear modulus in the seabed, use the wave equation for pressure in the water column and match solutions at the seabed interface, so that there is no loss of generality of the material here because the ocean dynamics discussions apply to both situations (fluid or elastic seabed).

\section{b. Methodologies}

Typical scenarios of sound propagation from one underwater point (the "source") to another (the "receiver") differ significantly from many familiar radiating wave applications, such as airborne radar or light (electromagnetic radiation). Refraction in the vertical plane can be very strong, and often it is the dominant kinematic wave effect. This strong refraction is one reason that ocean acoustics is frequently examined in a two-dimensional (2D) manner, in vertical $[r, z]$ planes extending outward from the geographic location of a point source. Slight azimuthal dependence of the environment is handled by allowing conditions to vary from plane to plane, the so-called $\mathrm{N} \times 2 \mathrm{D}$ modeling (see, for example, Kuperman et al. 1991). In this approximation, azimuthal energy flux is zero. This is fine for many applications, but there are many instances where this approach gives notably incorrect results, as illustrated in many papers (e.g., Oba and Finette 2002; Badiey et al. 2005; Lynch et al. 2010).

Fully $3 \mathrm{D}$ propagation methods, which allow sound to laterally refract away from the radials of $\mathrm{N} \times 2 \mathrm{D}$ models, are needed for accurate modeling in those situations. It must be emphasized that with accurate knowledge of the sound speed in any $3 \mathrm{D}$ ocean volume, 2D modeling can be done with a choice of established codes. Fewer implementations are available for 3D, among them parabolic equation (PE) one-way codes (Lin et al. 2013a; Heaney and Campbell 2016), coupled normal mode codes (Shmelev et al. 2014; Ballard et al. 2015), adiabatic (uncoupled) normal mode codes, and ray-based models such as Gaussian beams.

One specific application of the point-to-point analysis is ocean acoustic tomography (Munk et al., 1995), discussed further in Section 6. In the most developed version of this technology, sound travel time between distant points is used to map sound speed or temperature in a 3D volume over time, using sound transmitted from many sources to many receivers, as in medical tomography. For it to succeed, the paths that the sound takes must be known and the travel time carefully measured. Sometimes normal-mode travel times are used (horizontally refracting or straight paths), but usually rays refracting in a vertical plane are used. Single-path or few-path variants of this method use reduced amounts of equipment and target conditions along transects (in vertical planes) rather than volumes.

Full-field models can be used to model $p(x, y, z, t)$, needed as a starting point in linear inversion, but studies show that it is not necessary for tomography, with pulse travel-time analyzed in lieu of the full acoustic field. (The full field may be considered while estimating the travel-time and travel-time uncertainty (Flatté et al. 1979; Munk et al. 1995; Colosi 2016), but the travel times are used for oceanographic analysis and inversion.) 


\section{c. Propagation regimes and scattering regimes}

Sound in the temperate deep ocean can be trapped in the sound channel, which means that sound from deep sources can go long distances without encountering the seabed and surface. This most basic of propagation regimes, covered in the trio of books already cited (Flatté et al. 1979; Munk et al. 1995; Colosi 2016) is attractive to scientists for exploitation such as through tomography, but other marine acoustic operations do not have the flexibility to work around the boundary interactions and cannot use time averaging to reduce the impact of short-period internal waves (as tomography does) that impart a fluctuating nature to the sound.

In a typical stratified, shallow-water situation, fluctuating concave-downward sound-ray refraction, caused by warm upper-layer water with faster sound speed than underlying water, leads to repeated interaction with a complex seafloor. This, in turn, can lead to strong time-dependent sound fluctuations if evolving structures in the water column strongly perturb the seabed interactions, for example, structures found in nonlinear internal waves (Zhou et al. 1991; Preisig and Duda 1997; Duda and Preisig 1999; Oba and Finette 2002). (In that situation, knowing seafloor properties is as critical as knowing the nature of the refraction within the water if a fully quantitative result is desired, but here behavior of the water column is emphasized). Simultaneous fluctuating refraction in both the vertical and horizontal planes by nonlinear internal gravity waves (Apel et al. 2007) also can lead to temporally and spatially dependent attenuation, focusing, and multipath (Badiey et al. 2005; Lynch et al. 2010; Badiey et al. 2011; Duda et al. 2011a, 2012). These studies show that such waves can cause rapid and dramatic changes to sound fields in the ocean as the internal waves themselves propagate and evolve.

Many acoustical effects depend on frequency, and the detail required in a model can depend on the sound frequency band of interest. Not all frequencies travel far enough to benefit from detailed ocean state prediction. The salts in ocean water attenuate sound; the absorption coefficient essentially rises with the square of the frequency $\omega$ (Francois and Garrison, 1982). The net result is that sound capable of propagating more than a few tens of kilometers is limited to below about $15 \mathrm{kHz}$. This band corresponds to wavelengths $\lambda=2 \pi k^{-1}=2 \pi c \omega^{-1}$ greater than $10 \mathrm{~cm}$ (ocean sound speed varies over a range of about $\pm 2 \%$ from a nominal value of $c_{0}=1500 \mathrm{~m} / \mathrm{s}$ ). Sound propagation modeling that considers ocean and seabed variability is most often carried out for frequencies of 50 to $3000 \mathrm{~Hz}$, or $30 \mathrm{~m}>\lambda>0.5 \mathrm{~m}$.

As with most forward-scattering scenarios (i.e., propagation through random media scenarios), one can separate strong-scattering and weak-scattering problems. For weak scattering, where the intensity (squared amplitude) variance is less than 0.4 times the mean intensity squared (scintillation index $<0.4$ ), the Born approximation holds and the relationship between the scales of the sound-speed perturbations and the resulting scattered field is defined (e.g., Duda et al. 1988; Di Iorio and Farmer 1994) and can be exploited for measurement or data inversion purposes (Xu and Di Iorio 2011). This corresponds to the unsaturated fluctuation regime (Flatté et al. 1979; Colosi 2016). The Born approximation 
means that the incident field at all points from which the scattered field is computed is unchanged from the case of a uniform medium with no scattering (single scatter). On the other hand, under strong scattering sound can be drastically redirected, with the incident field significantly altered. Recent analysis of many ocean propagation scenarios tells us that strong scattering is expected in many situations, particularly for sound passing through strong nonlinear internal wave packets in shallow water (Badiey et al. 2005, 2011, 2013; Lynch et al. 2010; Duda et al. 2011a, 2012). A few images of fields undergoing strong scattering appear in Section 4. On the other hand, 3.5D propagation modeling of sound moving through internal tides (no short nonlinear waves) can show extended regions of weak scattering (Duda et al. 2013).

For weak scattering, to first order, waves propagating a distance $L$ are sensitive, near the middle of the propagation path, to refractive-index structures near the Fresnel zone scale length $R_{F}=(\lambda L)^{1 / 2}$ (Flatté et al. 1979; Farmer et al. 1987, Padmanabhan 2001), although this is only a guideline in strongly inhomogeneous media like the ocean (Rypina and Brown 2007). For the low-frequency band stated above, scales range from $R_{F}=31$ $\mathrm{m}$ (for $3 \mathrm{kHz}$ at $2 \mathrm{~km}$ ) to $R_{F}=1300 \mathrm{~m}$ (for $50 \mathrm{~Hz}$ at $35 \mathrm{~km}$ ). Weak-scattering models are usually spectral models, and statistical homogeneity of the heterogeneous medium is assumed and exploited. Of course, one can have weak scattering from a medium with variations of spatially-dependent statistics or time-dependent statistics, so really there are at least four combinations of strong or weak scattering and homogeneous or inhomogeneous statistics. For strong scattering, important patterns of sound focusing and shadowing (no sound) can develop from features of scales other than $R_{F}$. Notably, sound-speed-altering ocean phenomena such as waves passing between a source and receiver, or over a source (a special case of the above), have a variety of effects (Lin et al. 2009; Lynch et al. 2010; Duda et al. 2011a).

\section{d. Propagation simulation in the $4 D$-variable environment}

Ocean waves, ocean circulation (advection), and water mass evolution via mixing and boundary fluxes cause sound-speed fluctuations at scales larger than the acoustic wavelength and are the main drivers of acoustic fluctuations. The theory has been upheld by many of the aforementioned $2 \mathrm{D}, \mathrm{N} \times 2 \mathrm{D}$, and $3 \mathrm{D}$ simulations of sound propagation, and verified by measurements. A good example of propagation simulation thorough a complex ocean environment is available (Lermusiaux et al. 2010). These studies show slices through 4Dvariable sound-speed structures obtained from a data-driven ocean model, plus line drawings and histograms of sound level computed at points and along transects. Another example (Colin et al. 2013) also models sound using conditions obtained from an ocean model, and concentrates on time-dependence of the sound field along a single acoustic propagation path.

Lermusiaux et al. (2010) and Colin et al. (2013) use the most recent technology, simulating sound through environments computed with data-driven (data assimilating) ocean dynamical models (with one exception: they do not use 3D acoustic modeling). Stepping 
back from this level of complexity, which requires good data in four dimensions, other methods allow rudimentary propagation simulation and prediction. Geological databases and models, ocean circulation databases (atlases), and locally dense measurements also allow simulation, perhaps not of detailed sound fields but instead the statistics and the mean behavior of these effects. Using ocean dynamical model fields can improve these statistical predictions, but also may allow prediction of specific transient effects (deterministic prediction). However, making improvements to acoustic system performance prediction poses a strong challenge to ocean modeling capabilities. The reasons include incomplete dynamics in models (such as the absence of explicit, double-diffusive influences on intrusions known to affect sound (Colosi et al. 2012a; Duda and Sellers 2016)), insufficient resolution of the dynamical models, and insufficient data to constrain data-driven models in the absence of special regional focus. Data-constrained modeling is now routine (Edwards et al. 2015). However, most models barely resolve, incorrectly model, or exclude many features that have been shown to strongly influence sound propagation (see next section). These are internal tides, frontal features, bottom-boundary layer conditions, internal bores, and steep nonlinear internal waves (the last two factors feature nonhydrostatic pressure). Much recent research has demonstrated the strong acoustic effects of these processes, so the fact that they are currently beyond routine dynamical modeling present (challenging) opportunities for improvement. Note that both acoustic signal and noise modeling are covered implicitly in this discussion, because noise can be treated as an assemblage of signals.

\section{Illustrative example: sound in internal tidal waves}

Sound behavior in 4D-variable mesoscale ocean structures can be modeled with datadriven methods given adequate data (Lermusiaux et al. 2010; Edwards et al. 2015), but gravity-wave features that affect sound are more difficult to capture accurately with dynamical models. It is safe to say that all sound is influenced by internal waves, just as all undersea sound is influenced by local mesoscale conditions. Additionally, for deep-ocean propagation the sensitivity of the sound field (when broken into ray components) to perturbations from internal waves is variable with respect to location and depth because of ray geometries imparted by background (mesoscale-variable) stratification (Beron-Vera and Brown 2003). A subset of the complete spectrum of internal waves can be simulated with dynamical models that include tide-generating forces (best for large domains or the globe) or are forced by barotropic tidal signals at the boundaries. These can be executed in a data-driven mode (Lermusiaux et al. 2010) or in a mode with tidal forcing only (Ponte and Cornuelle 2013).

Example internal-wave perturbed acoustic fields that were computed using sound-speed fields taken from Southern California Bight simulations (Ponte and Cornuelle, 2013) are shown in Figures 1-3. Three-dimensional sound at $100 \mathrm{~Hz}$ is simulated using a Cartesian 3D split-step Fourier code (Lin et al. 2013a) within a volume of synthetic ocean environment 
ISN01HR00 $100.5 \mathrm{~m}$

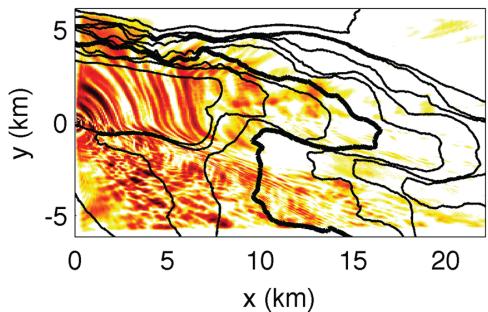

ISN01HR00 $250.5 \mathrm{~m}$

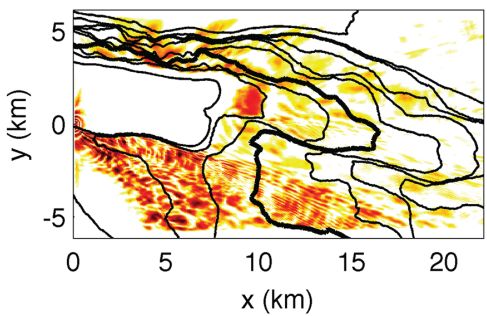

ISN01HR06 $1005 \mathrm{~m}$

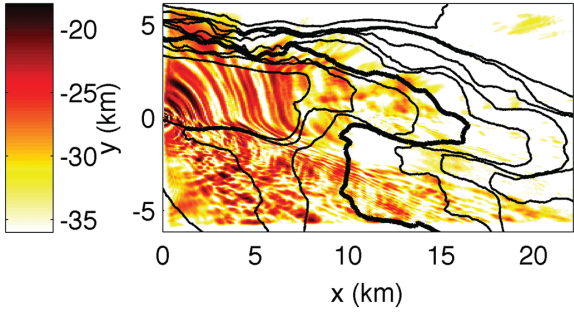

$-20$

$-25$

$-30$

$-35$

ISN01HR06 $250.5 \mathrm{~m}$
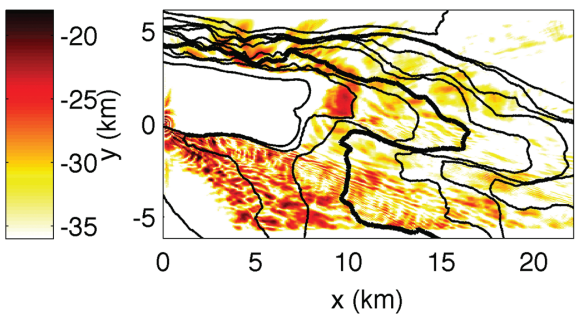

Figure 1. Simulated $100-\mathrm{Hz}$ sound level is shown in decibels (dB; with an arbitrary offset) for two horizontal planes (at 100 and $250 \mathrm{~m}$ depths) and at two different times, i.e., 6 hours apart. The source location (50 m depth at the $x, y$ origin) is west of Point Loma (San Diego, CA) by about $25 \mathrm{~km}$; the $x$ direction is northwest. The Coronado Escarpment and the deep San Diego Trough are at the top of the boxes. Depth contours are shown, 200- to 1200-m depths, 100-m interval; the $500-\mathrm{m}$ contour is thicker. The $\mathrm{dB}$ levels have been adjusted as a function of range to account for cylindrical spreading.
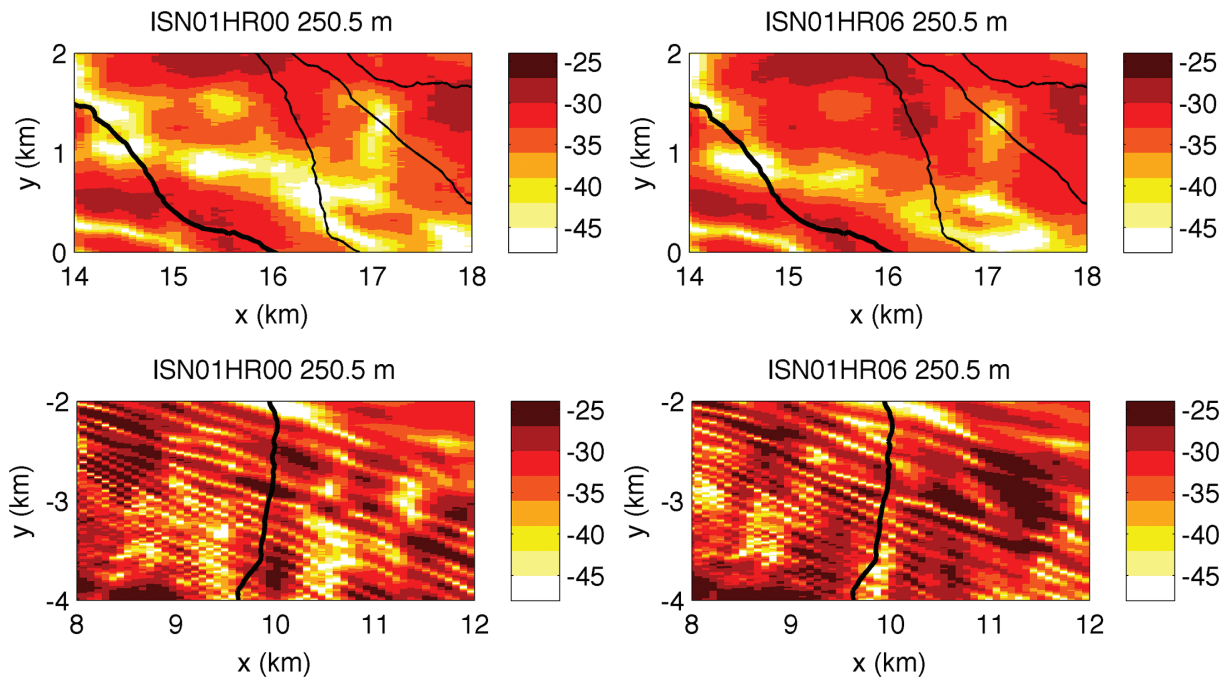

Figure 2. Data from the lower panel of Figure 1 are drawn for a pair of $2 \times 4-\mathrm{km}$ regions. The contour interval is three decibels (dbs). The color scale differs from that of Figure 1. 

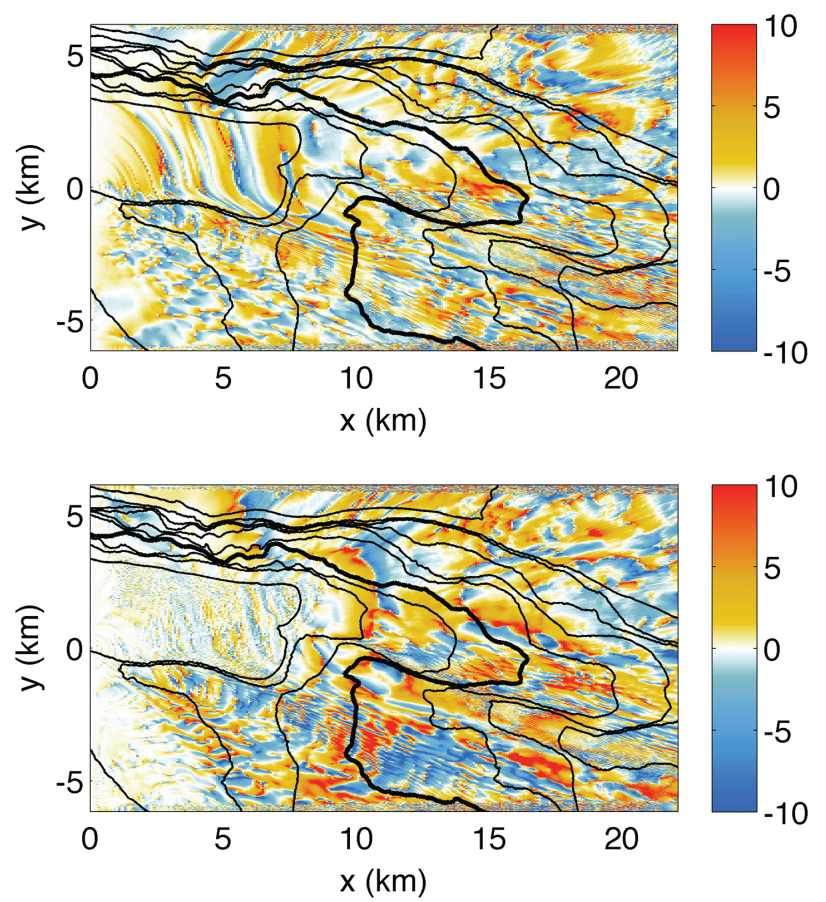

Figure 3. The differences of the fields (dB) of Figure 1 are shown for 100-m depth (top) and 250-m depth (bottom). Inside the shallowest (200-m) contour enclosing the area $0<y<4$ and $0<x<8$ $\mathrm{km}$, the 250-m depth sound is in the attenuating seabed and is very low level (see Fig. 1). There are kilometer-wide regions (or larger) where the sound level has changed dramatically in the 6-h interval with uniform sign, with changes occurring over the majority of the domain.

that develops from a layered starting condition by the action of the barotropic tidal boundary conditions. Figure 1 shows the intensity of sound at two times in two horizontal planes six hours apart. The ocean conditions differ considerably between the two simulations by the action of internal tides. The major patterns of sound may appear unchanged to the eye over time if the entire domain is casually examined, but the patterns are actually quite variable. Figure 2 shows expanded-scale views of the data of Figure 1, highlighting the strong differences.

In the 250-m depth-expanded plots (Fig. 2), there is visible movement in the $x$ direction of loud (high dB-level) regions that roughly follow lines in the $y$ direction. Along the $y$-directed high-level lines are short-scale patterns created by the interference of sound that is horizontally redirected by reflection from an escarpment and direct-path sound, a true 3D-propagation feature not included in $\mathrm{N} \times 2 \mathrm{D}$ modeling. (Details concerning the acoustic domain location and the reflection effect can be found in Duda et al. 2013.) Due in part to interference such as this, the data in Figure 2 are consistent with strong spatial 
variability of the correlation scale of the sound field (Duda et al. 2012, 2013), which has implications for sensing array performance (Finette and Oba 2003; Duda et al. 2012). Figure 3 shows the differences between the two fields at each level as seen in Figure 1. (Decibel differences are equivalent to ratios of power.) The images show power difference features over a broad range of scale lengths. Sometimes range-averaging algorithms are applied to fields, such as in Figures 1 and 2, to give results that are representative of what one would expect for a broadband acoustic signal (Harrison and Harrison 1995). Acoustic systems with broadfrequency bandwidth will show variation in the field spatial structure over the band, a fact that must be taken into account. A wide band of frequencies can be modeled and added coherently to achieve the desired is commonly used, is correct result, whereas the simpler range averaging (or depth averaging) approximates the desired result.

The internal tidal sound-speed anomalies (departures from a time-independent reference state) in this example have horizontal scale lengths of tens of kilometers, which are similar in scale to mesoscale-induced anomalies. The magnitude of the sound, field changes seen in the figures arguably also represents of what one may expect from an evolving mesoscale field, although sharp frontal boundaries and their associated submesoscale flow features might induce stronger sound-field effects, and mesoscale features alone would induce sound-field effects that evolve more slowly.

\section{Origins through current practice: knowing the ocean}

Recent advances in underwater sound propagation research mean that we are now oceanstate data-starved when it comes to our ability to predict how sound will behave in specific environments. The state of the water column is the subject here, but effects that are dependent on seabed properties are also important, and seabed conditions must be kept in mind in any acoustic modeling exercise. In addition to bathymetric uncertainty, there is a lack of knowledge, for many locations, of sub-bottom acoustic parameters that exert first-order control on sound propagation. For instance, seabed reflection behavior (amplitude and phase) are governed by conditions at and below the interface (Frisk 1994). Efforts to know seabed parameters are ongoing.

In the mid twentieth century, the newly invented bathythermograph, coupled with raybased (geometric optics regime) sound propagation models within vertical planes, allowed useful naval forecasting of sonar system performance. The propagation modeling was simple but powerful, and did not require 4D data, as it could not be used effectively. One might say that the acoustic modeling and the knowledge of the environment were well matched, although primitive by today's standards. Development of practical, full-wave computational propagation models late in the twentieth century (Robinson and Lee 1994; Jensen et al. 2011) allowed automated, reliable approaches to modeling sound in vertical planes, with a typical geometry consisting of radial planes meeting at an emitting source ( $\times 2 \mathrm{D}$ approximation). This innovation produced a demand for ocean data. Data collection systems (e.g., Davis et al. 1992; Greenslade et al. 1997; Le Traon and Dibarboure 1999) and ocean modeling 

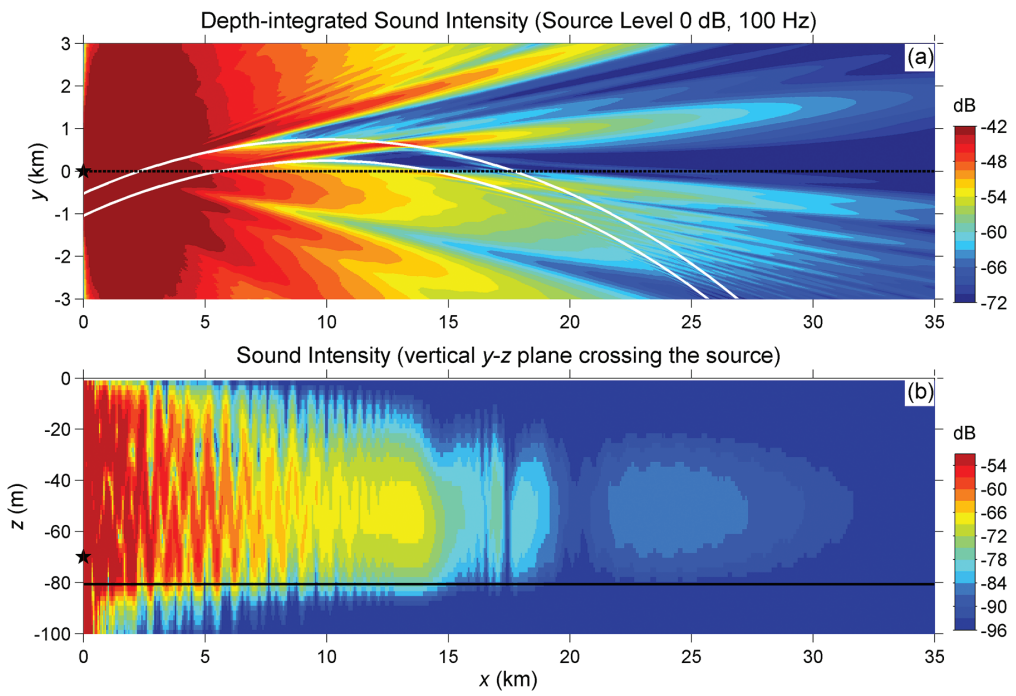

Figure 4. (a) Sound emitted from a 100-Hz source on the "outside" of a curved internal wave packet is plotted. Depth-integrated sound intensity is shown. The packet (two waves of depression, $40-\mathrm{km}$ radius of curvature, shown by the white lines) causes a complicated reflection, shadowing, and weak ducting pattern. (b) Sound intensity in the $y=0$ vertical slice, shown by the black line in (a), is plotted. (Decibel units are relative to source level unity. Reproduced from Lynch et al., 2010 with permission.)

methods (e.g., Marshall et al. 1997; Haidvogel et al. 2000) also advanced at that time. Yet in spite of these improvements, data assimilation was still being perfected (Robinson et al. 1998) and data were still sparse, and at this point the acoustic modeling capability surpassed environmental knowledge.

If the deep ocean is considered, there is now a good match between ocean knowledge and acoustic modeling, because $\mathrm{N} \times 2 \mathrm{D}$ modeling is sufficient, mesoscale fields can be incorporated from models, and small-scale internal wave effects can be computed statistically (Colosi 2016). However, $\mathrm{N} \times 2 \mathrm{D}$ modeling is not sufficient in areas where sound interacts with complex bathymetry, where water-mass boundaries such as tidal mixing fronts occur, and where strong nonlinear internal waves create intense lateral gradients of sound speed. Mostly, these are shallow-water areas. Figures 4 and 5 show some dramatic acoustic effects of realistically scaled internal waves in shallow water, with very strong localized focusing or shadowing of sound that would be uniform in the absence of internal waves in the ensonified area.

Currently, 3.5D full-wave propagation models, such as the one used in the previous section, are being refined to better model sound in environments where those features produce the so-called out-of-plane effects. (Note that a few good 3D studies 

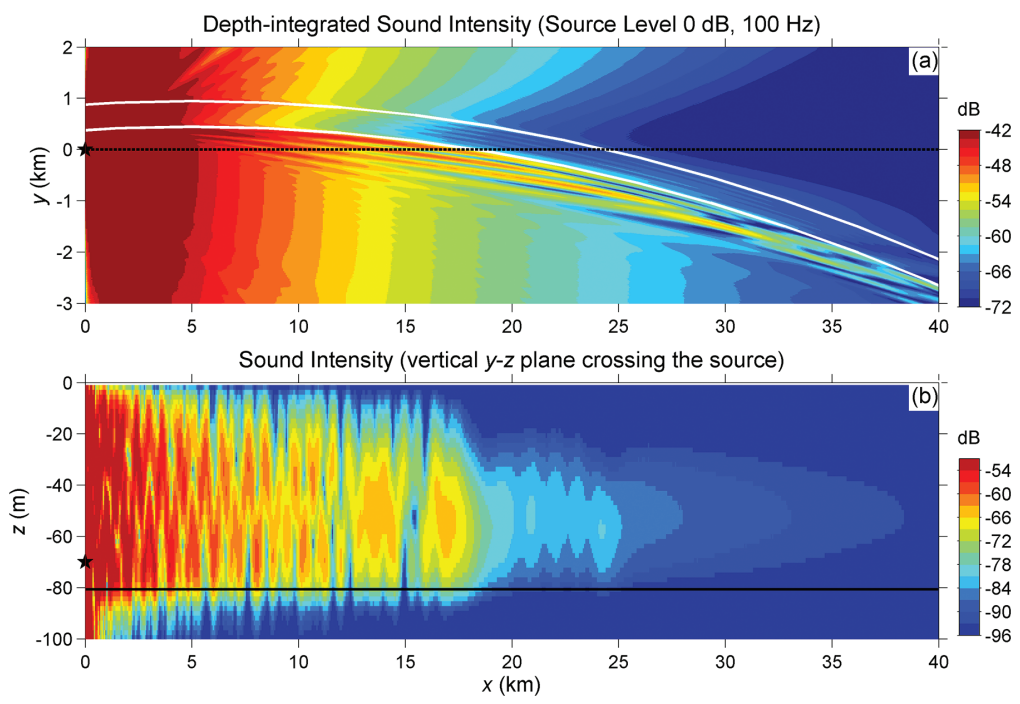

Figure 5. Simulation results similar to those of Figure 4 are shown. The $100-\mathrm{Hz}$ sound emitter is now in the "inside" of the curved wave packet, which has a 200-km radius of curvature. (a) A plan view of depth-averaged intensity is shown. Whispering gallery and shadowing effects are mainly seen. (b) Vertical slice of sound intensity along $y=0$. Mode one only weakly penetrates the duct, as seen at the right in (b). (Decibel units are relative to source level unity. Reproduced from Lynch et al., 2010 with permission.)

were made in the $\mathrm{N} \times 2 \mathrm{D}$ era.). Hence, $4 \mathrm{D}$-variable environmental input fields, if available, could be exploited in acoustic models. Because the contemporary ocean modeling and prediction capability is data-starved, as are most oceanographic endeavors, leveraging data with sparse sampling in space and time by infilling with data-constrained (data-assimilating (DA)) dynamical modeling (or state-space estimation) is a reasonable approach for efficiently building reliable 4D fields (Robinson et al. 1998; Edwards et al. 2015). Although this method is progressing for features that are resolved by the models, it is not yet possible for small-scale ocean features of first-order acoustic importance.

Lately, better measurement systems and evolving strategies have yielded many insights about the acoustic role of relatively small-scale submesoscale features and processes in the sea, such as the waves of Figures 4 and 5. Features evident in vertical plane dissections would be sharp fronts, thermohaline intrusions, and internal-wave displacements. Features evident in plan view would be fronts (again), filaments, eddies, and internal waves (again). Each of these features can have major impact on where sound energy goes in the ocean. Modeling them all faithfully in data-assimilating dynamical models would be a powerful capability, but is not reasonable at this time from the standpoints of computation and data collection. In particular, small-scale features exhibiting nonhydrostatic-pressure dynamics (Lamb 1994) 
and spatially-dependent or double-diffusive, subgrid-scale mixing are not currently included in DA models, although front predictability has received some recent attention (Jacobs et al. 2014). A reasonable alternative to full DA modeling of coastal nonlinear internal waves would the development of a prediction capability for key parameters, for example, peak amplitude, position, propagation direction, and quantity of waves in wave packets. Efforts are underway to augment data-constrained regional models by nesting internal-wave models and frontal-resolving models inside them for just this purpose (Duda et al. 2014a, b). This innovation may enable a meaningful internal-wave prediction-then acoustic predictioncapability.

\section{Deterministic, statistical, and analytic feature models}

The preceding sections suggest that, given sufficient knowledge of the 4D ocean environment, tools are available to model sound propagation with enough accuracy to obtain useful information. The acoustic effects of large-scale features are approachable using data-constrained deterministic ocean modeling and deterministic acoustic modeling (see example in Section 3, although it did not incorporate ocean data), but such modeling would be insufficient for those features at the smallest scales. A supplemental approach to deterministic modeling is to employ statistical models for the small-scale ocean features, from which acoustic field statistics can be derived. Some deterministic, statistical, and analytic methodologies for joint dynamical and acoustical modeling are summarized in a list below.

Before moving to the list of linked dynamical and acoustical model methods, dynamical ocean modeling is considered. The most comprehensive (but data-hungry) method for deterministic ocean modeling is DA dynamical modeling. The advantage of this method is that it allows the optimal use of all available data. Shortfalls in quantity and quality of data present fundamental limitations in any implementation. More generally, the (typical) hydrostaticpressure assumption, required for reasonable computing speed, disallows internal tidal bores and energetic, short internal waves. Resolution limits and lack of double-diffusive mixing may inhibit accuracy of water-mass front predictions. Understanding and working around these limits of modern DA models is at the forefront of predictive ocean acoustics, for example, in cataloging uncertainty (Lermusiaux et al. 2010). Note that methods to infill DA-modeled areas with nonhydrostatic internal waves are currently being examined for accuracy (Duda et al. 2014b).

\section{a. Deterministic ocean model linked to deterministic acoustics model}

In an environment where acoustic field features are largely determined by ocean features resolved by dynamical models (DA or otherwise), one can simply use the $c(x, y, z, t)$ field of a model for an acoustic computation. Computations can be 2D (vertical slice), 3D, or 3.5D. The results can be used to study coupling of specific acoustic and ocean features and to map acoustic energy within the computational domain. They can also be used to extract 
statistics such as spatial coherence and principal components of the $4 \mathrm{D}$ variable field, to be input to statistical models.

\section{b. Deterministic ocean model linked to statistical acoustics model}

The statistics of acoustic fields can be obtained by the approach described above, but some sound field statistics can be computed from the statistics of the environment, i.e., by treating the small details of the environment stochastically (Flatté et al. 1979; Colosi 2016). Example sound statistics are intensity and phase variances, and field correlation scales. The necessary statistics of the environment can be obtained by analyzing output of dynamical models, and may include RMS sound-speed variations, correlation scales of those variations, or mode coupling matrices (Colosi and Morozov 2009; Colosi et al. 2012b, 2013; Raghukumar and Colosi 2014). One aspect of this approach is that one can treat the ocean as non-stationary and examine the evolution of statistical quantities. However, this approach has not been used as often as method \#3, below.

\section{c. Statistical ocean model and statistical acoustics model}

Some ocean phenomena can be reasonably well described by empirical statistical models derived from data, such as deep-ocean internal waves (Levine 2002). Ensembles of dynamical ocean models that are properly formulated for specific dynamical processes can also be used to define the statistics of those processes. Statistics of the acoustic field can be computed and then examined by feeding the ocean statistics (model- or data-derived) into statistical acoustic models (Flatté et al. 1979; Colosi and Morozov 2009; Colosi et al. 2012b, 2013; Gong et al. 2013; White et al. 2013; Raghukumar and Colosi 2014; Rouseff and Lunkov 2015; Colosi 2016).

\section{d. Statistical ocean model and deterministic acoustics model}

Ensembles of deterministic acoustic simulations can be made with dynamical ensemble members, yielding statistics. It is also possible to examine sound propagation behavior in specific ocean structures, but at this time this action is done more often with the method described in \#5.

\section{e. Analytic feature model and acoustics model}

For process studies and rough-estimate modeling, analytic descriptions of ocean dynamical features may suffice and may be better suited to process studies, for example, a study relating parameterized front curvature to sound focusing. Figures 4 and 5 illustrate fields used in process studies of sound in isolated strong internal waves. Process studies, showing interesting results regarding the structure of ducted and redirected sound energy, are numerous (Badiey et al. 2011; McMahon et al. 2012; Lin et al. 2013b). 


\section{Model-based acoustic simulation in practice}

Although meaningful deterministic predictive modeling of sound in the actual conditions of the 4D-variable ocean may be beyond the current state of the art in regions with important submesoscale features (see Section 4), there are many scenarios in which models are capable of providing useful input for ocean acoustic predictions. Here are four well-established protocols showing modeled sound-speed fields used in acoustic predictions.

1. Ocean acoustic tomography (OAT) and ocean climate thermometry (OCT) signal prediction

2. Random fluctuation prediction for OAT and OCT (noise component of signal)

3. Signal-to-noise ratio prediction (signal and noise modeling)

4. Acoustic coherence prediction for signal processing

The first of these protocols is covered fully in the book by Munk et al. (1995), and later papers (Dushaw et al. 2009, 2013; Dushaw, 2013). In brief, sound is transmitted between the many elements of a horizontal array of equipment spanning tens to thousands of kilometers. The arriving signals (times of multipath pulse arrivals, essentially) are used to map the sound-speed structure in the intervening medium. Ocean acoustic tomography uses a dense array and overlapping paths to generate a 3-D volumetric result, whereas ocean climate thermometry is less comprehensive. Thermometry may be as targeted as recording the path-averaged sound speed along a single geodesic path (close to the path-averaged temperature). Although spatially limited when compared to a tomography result, the value of a long thermometry time series can be high. Note that in a single path the multipath arrival times are approximated by ray trajectories in the plane-like $(r, z)$ surface, where $r$ is distance long the geodesic path. Ocean acoustic tomography projects have demonstrated that a linearized inverse method of obtaining the sound-speed character from data can work well for tomography. The linearized method depends on a good a priori model for sound-speed. Climatology has proven to be an adequate a priori model in many cases (AMODE-MST Group 1994; Dushaw et al. 1996); dynamical ocean models can extend the usability of the linearized method to more challenging regions.

The tomography methodology has been used most often for long-distance propagation at mid latitudes, where sound is ducted (with respect to depth) about the sound-speed minimum, which is at order 1000-km depth (i.e., sound is trapped in the sound fixing and ranging (SOFAR) channel or sound channel). The studies have shown that ray tracing in vertical planes (an $\mathrm{N} \times 2 \mathrm{D}$ method) is adequate to define the paths over which the sound moves, enabling inversion, shown early on by Spiesberger et al. (1980), and with great detail in the context of a traveling-source inverse study by Dushaw et al. (1996). This ray model can be extended to take into account the finite volumes surrounding rays to which sound is coherently sensitive (Skarsoulis et al. 2009), a more detailed treatment than considering sound to travel along infinitesimal rays. Ocean waveguide acoustic normal modes speeds are 
sometimes used for tomographic purposes (Heaney et al. 1991; Dushaw and Menemenlis 2014). In this procedure, ocean dynamical models (or climatological models) are used to compute horizontal modal ray paths and modal travel times along the paths, with the paths showing refraction from gradients in the ocean conditions. Modal tomography is thus another procedure to use acoustic information to constrain conditions in large volumes of ocean instantaneously and repeatedly. Note that horizontal refraction of rays or modes can be disregarded on many situations: the references (Heaney et al. 1991; Dushaw and Menemenlis 2014) pertain to sound that travels one-half way around Earth, a special situation that must consider horizontal refraction, and the strongly bottom-interacting effects illustrated in Section 3 can be avoided in tomographic exercises.

Ocean acoustic tomography and OCT require filtering of signals to separate the timing fluctuations that are caused by internal waves (noise) from the steady or slowly varying timing anomalies that are caused by mappable mesoscale features (signal). The GarrettMunk spectral model of ocean internal waves has been used to model the fluctuations (Flatté et al. 1979) for the purposes of experiment design (see Chapter 5 in Munk et al.) and a data averaging protocol prior to inversion (Cornuelle et al. 1993).

The prediction of signal strength as a function of range, bearing, and depth with respect to a known underwater sound source is the subject of a vast literature. This subject is often analyzed in terms of transmission loss (TL), measured in decibels relative to the source level. Example papers, chosen from hundreds, are Ngheim-Phu and Tappert (1985), Finette et al. (2007), and the more recent Colin et al. (2013). The technique of modeling noise (sound from distributed sources) can be done by adding fields from many sources (Kuperman and Ingenito 1980; Udovydchenkov et al. 2010), Together, these enable signal-to-noise ratio modeling and via the spatial coherences of the modeled signal and noise, they enable modeling of coherent array processing gain (Finette and Oba 2003; Real et al. 2015).

\section{Conclusion}

The ocean acoustic predictive goal of knowing sound-field details in identified regions of interest, by virtue of sufficiently knowing the ocean, is attainable to a degree that is evolving. Methods continue to develop; many tools are in place and more are being developed. It is reasonable to say that long-range sound propagation studies and forecasts, which by nature include only the most robust acoustic features, benefit more from dynamical model state predictions than do short-range studies. Sometimes a few measurements can adequately determine the conditions in short-range studies, although sometimes not. As far as propagation simulations go, the speed of full-wave modeling (as distinguished from ray modeling) varies inversely with frequency (in cubic fashion for 3D), so volumetric field predictions that include aspects of temporal and spatial coherency, as well as signal strength, are most reasonable for low frequencies. Serendipitously, sound energy at high frequency is absorbed by seawater, so reasonably fast, long-distance propagation simulations covering 20 to 1000 $\mathrm{Hz}$ are often sufficient to describe the majority of energy. Interestingly, this frequency band 
is used by many marine mammals, and they may exploit the long propagation ranges. Their hypothesized ability to form signals that counteract the complex reverberation and complex, forward-scattered propagation would be notable if true. Conversely, their vocalization and hearing may provide them a view of their environment that is shaped by the acoustic filtering effects of the processes we investigate with linked ocean and acoustic models.

Acknowledgments. Funding for this work was provided by the U.S. Office of Naval Research, Ocean Acoustics Program, Grants N-00014-11-1-0701 and N00014-14-1-0223. Discussions with Bruce Cornuelle, John Colosi, Ying-Tsong Lin, James Lynch, Arthur Newhall, Gordon Zhang, Brian Dushaw, and Pierre Lermusiaux have been valuable and are appreciated.

\section{REFERENCES}

AMODE-MST (Acoustic Mid-Ocean Dynamics Experiment) Group. 1994. Moving ship tomography in the North Atlantic. Eos 75(2), 17-23 doi: 10.1029/94EO00509

Anderson, D. M., A. D. Cembella, and G. M. Hallegraeff. 2012. Progress in understanding harmful algal blooms: paradigm shifts and new technologies for research, monitoring, and management. Ann. Rev. Mar. Sci., 4, 143-176. doi: 10.1146/annurev-marine-120308-081121

Apel, J. R., L. A. Ostrovsky, Y. A. Stepanyants, and J. F. Lynch. 2007. Internal solitons in the ocean and their effect on underwater sound. J. Acoust. Soc. Am., 121, 695-722 doi: 10.1121/1.2395914

Badiey, M., B. G. Katsnelson, J. F. Lynch, S. Pereselkov, and W. L. Siegmann. 2005. Measurement and modeling of three-dimensional sound intensity variations due to shallow-water internal waves. J. Acoust. Soc. Am., 117, 613-625. doi: 10.1121/1.3553374

Badiey, M., B. G. Katsnelson, Y.-T. Lin, and J. F. Lynch. 2011. Acoustic multipath arrivals in the horizontal plane due to approaching nonlinear internal waves. J. Acoust. Soc. Am., 129, EL141EL147. doi: 10.1121/1.3553374

Badiey, M., L. Wan, and A. Song. 2013. Three-dimensional mapping of evolving internal waves during the Shallow Water 2006 experiment. J. Acoust. Soc. Am., 134, EL7-EL13. doi: 10.1121/1.4804945

Ballard, M. S., B. M. Goldsberry, and M. J. Isakson. 2015. Normal mode analysis of threedimensional propagation over a small-slope cosine shaped hill. J. Comput. Acoust., 23, 1550005. doi: 10.1142/S0218396X15500058

Beron-Vera, F. J., and M. G. Brown. 2003. Ray stability in weakly range-dependent sound channels. J. Acoust. Soc. Am., 114, 123-130. doi: 10.1121/1.1582444

Brekhovskikh, L. M., and Y. P. Lysanov. 2001. Fundamentals of Ocean Acoustics, $3^{\text {rd }}$ ed. AIP Series in Modern Acoustics and Signal Processing. New York: AIP/Springer-Verlag. 280 pp.

Brewer, P. G., D. M. Glover, C. Goyet and D. K. Shafer. 1995. The pH of the North Atlantic Ocean: improvements to the global model for sound absorption. J. Geophys. Res. Oceans, 100, 8761-8776. doi: 10.1029/95JC00306

Colin, M. E. G. D., T. F. Duda, L. A. te Raa, T. van Zon, P. J. Haley, P. F. J. Lermusiaux et al. 2013. Timeevolving acoustic propagation modeling in a complex ocean environment. In Oceans '13, Bergen Conference Proceedings, IEEE/MTS. Red Hook, NY: Curran Associates. doi: 10.1109/OCEANSBergen.2013.6608051

Collins, M. D. 1991. Higher-order Padé approximations for accurate and stable elastic parabolic equations with application to interface wave propagation. J. Acoust. Soc. Am., 89, 1050-1057. doi: $\underline{10.1121 / 1.400646}$ 
Collins, M. D., W. A. Kuperman, and W. L. Siegmann. 1995. A parabolic equation for poro-elastic media. J. Acoust. Soc. Am., 98, 1645-1656. doi: 10.1121/1.413431

Collins, M. D., J. F. Lingevitch, and W. L. Siegmann. 1997. Wave propagation in poro-acoustic media. Wave Motion, 25, 265-272. doi: 10.1016/S0165-2125(96)00045-5

Collis, J. M., W. L. Siegmann, F. B. Jensen, M. Zampolli, E. T. Küsel, and M. D. Collins. 2008. Parabolic equation solution of seismo-acoustics problems involving variations in bathymetry and sediment thickness. J. Acoust. Soc. Am., 123, 51-55. doi: 10.1121/1.2799932

Colosi, J., A. 2016. Sound Propagation Through the Stochastic Ocean. Cambridge: Cambridge University Press. $424 \mathrm{pp}$.

Colosi, J. A., and A, K. Morozov. 2009. Statistics of normal mode amplitudes in an ocean with random sound-speed perturbations: Cross-mode coherence and mean intensity. J. Acoust. Soc. Am., 126, 1026-1035. doi: 10.1121/1.3158818

Colosi, J. A., T. F. Duda, Y.-T. Lin, J. F. Lynch, A. E. Newhall, and Bruce D. Cornuelle. 2012a. Observations of sound-speed fluctuations on the New Jersey continental shelf in the summer of 2006. J. Acoust. Soc. Am., 131, 1733-1748. doi: 10.1121/1.3666014

Colosi, J. A., T. F. Duda, and A. K. Morozov. 2012b. Statistics of low-frequency normal-mode amplitudes in an ocean with random sound-speed perturbations: Shallow-water environments. J. Acoust. Soc. Am., 131, 1749-1761. doi: 10.1121/1.3666002

Colosi, J. A., T. K. Chandrayadula, A. G. Voronovich, and V. E. Ostashev. 2013. Coupled mode transport theory for sound transmission through an ocean with random sound speed perturbations: coherence in deep water environments. J. Acoust. Soc. Am., 134, 3119-3133. doi: 10.1121/1.4818779

Cornuelle, B. D., P. F. Worcester, J. A. Hildebrand, W. S. Hodgkiss, Jr., T. F. Duda, J. Boyd et al. 1993. Ocean acoustic tomography at $1000-\mathrm{km}$ range using wavefronts measured with a large aperture vertical array. J. Geophys. Res. Oceans, 98, 16365-16377. doi: 10.1029/93JC01246

Davis, R. E., D. C. Webb, L. A. Regier and J. Dufour. 1992. The Autonomous Lagrangian Circulation Explorer (ALACE). J. Atmos. Ocean. Technol., 9, 264-285. doi: 10.1175/1520-0426(1992)009< 0264:TALCE > 2.0.CO;2

Deane, G. B., J. C. Preisig, James C., C. T. Tindle, A. Lavery, and M. D. Stokes. 2012. Deterministic forward scatter from surface gravity waves. J. Acoust. Soc. Am., 132, 3673-3686. doi: 10.1121/ 1.4763996

Del Grosso, V. A. 1974. New equation for the speed of sound in natural waters (with comparisons to other equations). J. Acoust. Soc. Am., 56, 1084-1091. doi: 10.1121/1.1903388

Di Iorio, D., and D. M. Farmer. 1994. Path-averaged turbulent dissipation measurements using highfrequency acoustical scintillation analysis. J. Acoust. Soc. Am., 96, 1056-1069. doi: 10.1121/ 1.410382

Duda, T. F., and J. C. Preisig. 1999. A modeling study of acoustic propagation through moving shallow-water solitary wave packets. IEEE J. Ocean. Eng., 24, 16-32. doi: 10.1109/48.740153

Duda, T. F., and C. J. Sellers. 2016. Microscale, finescale, and mesoscale measurements made during the 2004 Structured Mixing Project (Micro-Tow 04) cruise. WHOI Tech. Rep. WHOI-2016-02. Woods Hole, MA: Woods Hole Oceanographic Institution. doi: 10.1575/1912/8007

Duda, T. F., S. M. Flatté, and D. B. Creamer. 1988. Modelling meter-scale acoustic intensity fluctuations from oceanic fine structure and microstructure. J. Geophys. Res. Oceans, 93, 5130-5142. doi: 10.1029/JC093iC05p05130

Duda, T. F., Y.-T. Lin, and D. B. Reeder. 2011a. Observationally constrained modeling of sound in curved ocean internal waves: examination of deep ducting and surface ducting at short range. J. Acoust. Soc. Am., 130, 1173-1187. doi: 10.1121/1.3605565 
Duda, T. F., Y.-T. Lin, W. Zhang, B. D. Cornuelle, and P. F. J. Lermusiaux. 2011b. Computational studies of three-dimensional ocean sound fields in areas of complex seafloor topography and active ocean dynamics. In Proceedings of 10th International Conference on Theoretical and Computational Acoustics, ICTCA 2011. Taipei, World Scientific Publishing.

Duda, T. F., J. M. Collis, Y.-T. Lin, A. E. Newhall, J. F. Lynch, and H. A. DeFerrari. 2012. Horizontal coherence of low-frequency fixed-path sound in a continental shelf region with internal-wave activity. J. Acoust. Soc. Am., 131, 1782-1797. doi: 10.1121/1.3666003

Duda, T. F., Y.-T. Lin, and B. D. Cornuelle. 2013. Scales of time and space variability of sound fields reflected obliquely from underwater slopes. J. Acoust. Soc. Am., 133, 3346. doi: 10.1121/ 1.4805665

Duda, T. F., Y.-T. Lin, A. E. Newhall, K. R. Helfrich, W. G. Zhang, M. Badiey et al. 2014a. The "Integrated Ocean Dynamics and Acoustics" (IODA) hybrid modeling effort. Proceedings of the 2nd International Underwater Acoustics - 2014 (UA2014), 621-628. UA2014 - $2^{\text {nd }}$ International Conference and Exhibition on Underwater Acoustics.

Duda, T. F., W. F. Zhang, K. R. Helfrich, A. E. Newhall, Y.-T. Lin, J. F. Lynch et al. 2014b. Issues and progress in the prediction of ocean submesoscale features and internal waves. OCEANS'14 MTS/IEEE. Washington, D.C.: Marine Technology Society.

Dushaw, B. D. 2013. Ocean acoustic tomography. In Encyclopedia of Remote Sensing, E. G. Njoku, ed. New York: Springer-Verlag.

Dushaw, B. D., and D. Menemenlis. 2014. Antipodal acoustic thermometry: 1960, 2004. Deep Sea Res., Part I. 86, 1-20. doi: 10.1016/j.dsr.2013.12.008

Dushaw, B. D., P. F. Worcester, and B. D. Cornuelle. 1993. On equations for the speed of sound in seawater. J. Acoust. Soc. Am., 93, 255-275. doi: 10.1121/1.405660

Dushaw, B. D., P. F. Worcester, B. D. Cornuelle, A. R. Marshall, and B. M. Howe. 1996. Data Report: Acoustic Mid-Ocean Dynamics Experiment (AMODE). Tech. Memo. APL-UW TM2-96. Seattle: University of Washington Applied Physics Lab. 278 p.

Dushaw, B. D., P. F. Worcester, W. H. Munk, R. C. Spindel, J. A. Mercer, B. M. Howe et al. 2009. A decade of acoustic thermometry in the North Pacific Ocean, J. Geophys. Res. Oceans, 114, C07021, doi: 10.1029/2008JC005124

Dushaw, B. D., P. F. Worcester, M. A. Dzieciuch, and D. Menemenlis. 2013. On the time-mean state of ocean models and the properties of long range acoustic propagation. J. Geophys. Res. Oceans, 118, 4346-4362. doi: 10.1002/jgrc.20325

Edwards, C. A., A. M. Moore, I. Hoteit, and B. D. Cornuelle. 2015. Regional ocean data assimilation. Ann. Rev. Mar. Sci, 7, 21-42. doi: 10.1146/annurev-marine-010814-015821

Farmer, D. M., S. F. Clifford, and J. A. Verrall. 1987. Scintillation structure of a turbulent tidal flow. J. Geophys. Res. Oceans, 92, 5369-5382. doi: 10.1029/JC092iC05p05369

Finette, S., and R. Oba. 2003. Horizontal array beamforming in an azimuthally anisotropic internal wave field. J. Acoust. Soc. Am., 114, 131-144. doi: 10.1121/1.1582441

Finette, S., R. Oba, C. Shen, and T. Evans. 2007. Acoustic propagation under tidally driven, stratified flow. J. Acoust. Soc. Am., 121, 2575-2590. doi: 10.1121/1.2713724

Flatté, S. M., R. Dashen, W. H. Munk, K. M. Watson, and F. Zachariasen. 1979. Sound Transmission through a Fluctuating Ocean. Cambridge: Cambridge University Press. 320 p.

Francois, R. E., and G. R. Garrison. 1982. Sound absorption based on ocean measurements. Part II: Boric acid contribution and equation for total absorption. J. Acoust. Soc. Am., 72, 1879-1890, doi: $10.1121 / 1.388673$ 
Frisk, G. V. 1994. Ocean and Seabed Acoustics: A Theory of Wave Propagation. Upper Saddle River, NJ: PTR Prentice-Hall. 300 p.

Gong, Z., T. Chen, P. Ratilal, and N. C. Makris. 2013. Temporal coherence of the acoustic field forward propagated through a continental shelf with random internal waves. J. Acoust. Soc. Am., 134, 3476-3485, doi: 10.1121/1.4824157

Greenslade, D. J. M., D. B. Chelton, and M. G. Schlax. 1997. The midlatitude resolution capability of sea level fields constructed from single and multiple satellite altimeter datasets. J. Atmos. Ocean. Technol., 14, 849-870. doi: 10.1175/1520-0426(1997)014<0849:TMRCOS > 2.0.CO;2

Haidvogel, D. B., J. Blanton, J. C. Kindle, and D. R. Lynch. 2000. Coastal ocean modeling: processes and real-time systems. Oceanography, 13, 35-46. doi: 10.5670/oceanog.2000.51

Harrison, C. H., and J. A. Harrison. 1995. A simple relationship between frequency and range averages for broadband sonar. J. Acoust. Soc. Am., 97, 1314-1317. doi: 10.1121/1.412172

Heaney, K. D., and R. L. Campbell. 2016. Three-dimensional parabolic equation modeling of mesoscale eddy deflection. J. Acoust. Soc. Am., 139, 918-926. doi: 10.1121/1.4942112

Heaney, K. D., W. A. Kuperman, and B. E. McDonald. 1991. Perth-Bermuda sound propagation (1960): Adiabatic mode interpretation. J. Acoust. Soc. Am., 90, 2586-2594. doi: 10.1121/1.402062

Jacobs, G. A., J. G. Richman, J. D. Doyle, P. L. Spence, B. P. Bartels, C. N. Barron et al. 2014. Simulating conditional deterministic predictability within ocean frontogenesis. Ocean Model., 78, 1-16. doi: 10.1016/j.ocemod.2014.02.004

Jensen, F. B., W. A. Kuperman, M. B. Porter, and H. Schmidt. 2011. Computational Ocean Acoustics; 2nd ed. New York: Springer-Verlag. 794 pp.

Kuperman, W. A., and F. Ingenito. 1980. Spatial correlation of surface generated noise in a stratified ocean. J. Acoust. Soc. Am., 67, 1988-1996. doi: 10.1121/1.384439

Kuperman, W. A., M. B. Porter, J. S. Perkins, and R. B. Evans. 1991. Rapid computation of acoustic fields in three-dimensional ocean environments. J. Acoust. Soc. Am., 89, 125-133. doi: 10.1121/ 1.400518

Lamb, K.1994. Numerical experiments of internal wave generation by strong tidal flow across a finite amplitude bank edge. J. Geophys. Res. Oceans, 99, 843-864. doi: 10.1029/93JC02514

Lermusiaux, P. F. J., J. Xu, C.-F. Chen, S. Jan, L. Y. Chiu, and Y.-J. Yang. 2010. Coupled oceanacoustic prediction of transmission loss in a continental shelfbreak region: Predictive skill, uncertainty quantification, and dynamical sensitivities. IEEE J. Ocean. Eng., 35, 895-916. doi: 10.1109/ JOE.2010.2068611

Le Traon, P. Y., and G. Dibarboure. 1999. Mesoscale mapping capabilities of multiple-satellite altimeter missions. J. Atmos. Ocean. Technol., 16, 1208-1223. doi: 10.1175/1520-0426(1999)016<1208: MMCOMS $>2.0 . \mathrm{CO} ; 2$

Levine, M. D. 2002. A modification of the Garrett-Munk internal wave spectrum. J. Phys. Oceanogr., 32, 3166-3181. doi: 10.1175/1520-0485(2002)032<3166:AMOTGM > 2.0.CO;2

Lin, Y.-T., T. F. Duda, and J. F. Lynch. 2009. Acoustic mode radiation from the termination of a truncated nonlinear internal gravity wave duct in a shallow ocean area. J. Acoust. Soc. Am., 126, 1752-1765. doi: 10.1121/1.3203268

Lin, Y.-T., T. F. Duda and A. E. Newhall. 2013a. Three-dimensional sound propagation models using the parabolic-equation approximation and the split-step Fourier method. J. Comput. Acoust., 21, 1250018. doi: 10.1142/S0218396X1250018X

Lin, Y.-T., K. G. McMahon, J. F. Lynch, and W. L. Siegmann. 2013b. Horizontal ducting of sound by curved nonlinear internal gravity waves in the continental shelf areas. J. Acoust. Soc. Am., 133, 37-49. doi: $\underline{10.1121 / 1.4770240}$ 
Lin, Y.-T., T. F. Duda, C. Emerson, G. Gawarkiewicz, A. E. Newhall, B. Calder et al. 2015. Experimental and numerical studies of sound propagation over a submarine canyon northeast of Taiwan, IEEE J. Ocean. Eng., 40, 237-249. doi: 10.1109/JOE.2013.2294291

Lynch, J. F., Y.-T. Lin, T. F. Duda, and A. E. Newhall. 2010. Acoustic ducting, reflection, refraction, and dispersion by curved nonlinear internal waves in shallow water. IEEE J. Ocean. Eng., 35, 12-27. doi: 10.1109/JOE.2009.2038512

Marshall, J., A. Adcroft, C. Hill, L. Perelman, and C. Heisey. 1997. A finite-volume, incompressible Navier Stokes model for studies of the ocean on parallel computers. J. Geophys. Res. Oceans, 102, 5753-5766. doi: 10.1029/96JC02775

McMahon, K. G., L. K. Reilly-Raska, W. L. Siegmann, J. F. Lynch, and T. F. Duda. 2012. Horizontal Lloyd mirror patterns from straight and curved nonlinear internal waves. J. Acoust. Soc. Am., 131, 1689-1700. doi: 10.1121/1.3666004

Munk, W. H., P. Worcester, and C. Wunsch. 1995. Ocean Acoustic Tomography. Cambridge: Cambridge University Press. 433 pp.

Nghiem-Phu, L., and F. Tappert. 1985. Parabolic equation modeling of the effects of ocean currents on sound transmission and reciprocity in the time domain. J. Acoust. Soc. Am., 78, 642-648. doi: $10.1121 / 1.392432$

Oba, R., and S. Finette. 2002. Acoustic propagation through anisotropic internal wave fields: transmission loss, cross-range coherence, and horizontal refraction. J. Acoust. Soc. Am., 111, 769-784. doi: $10.1121 / 1.1434943$

Padmanabhan, T. 2001. Theoretical Astrophysics, Vol. 2: Stars and Stellar Systems. Cambridge: Cambridge University Press. 575 pp. (Chapter 6)

Ponte, A. L., and Cornuelle. B. D., 2013. Coastal numerical modelling of tides: Sensitivity to domain size and remotely generated internal tide. Ocean Model., 62, 17-26. doi: 10.1016/j.ocemod. 2012.11.007

Preisig, J. C., and T. F. Duda. 1997. Coupled acoustic mode propagation through continental shelf internal solitary waves. IEEE J. Ocean. Eng., 22, 256-269. doi: $10.1109 / 48.585945$

Raghukumar, K., and J. A. Colosi. 2014. High frequency normal mode statistics in a shallow water waveguide: The effect of random linear internal waves. J. Acoust. Soc. Am., 136, 66-79. doi: $10.1121 / 1.4881926$

Real, G., X. Cristol, D. Habault, J.-P. Sessarego, and D. Fattaccioli. 2015. Influence of de-coherence effects on sonar array gain: Scales experiment, simulations and simplified theory comparison. UACE2015; $3^{\text {rd }}$ Underwater Acoustics Conference \& Exhibition, June 2015, Chania, Greece. $<$ hal-01313631>

Robinson, A. R., and D. Lee (edited by and with an introduction by). 1994. Oceanography and Acoustics: Prediction and Propagation Models. New York: AIP Press. 258 pp.

Robinson, A. R., P. F. J. Lermusiaux, and N. Q. Sloan III. 1998. Data Assimilation. In The Sea, Ideas and Observations on Progress in the Study of the Seas. Vol. 10. The Global Coastal Ocean. Processes and Methods. New York: John Wiley and Sons.

Rouseff, D., and A. A. Lunkov. 2015. Modeling the effects of linear shallow-water internal waves on horizontal array coherence. J. Acoust. Soc. Am., 138, 2256-2265. doi: 10.1121/1.4930954

Rypina, I. I., and M. G. Brown. 2007. On the width of a ray. J. Acoust. Soc. Am., 122, 1440-1448. doi: $10.1121 / 1.2764465$

Shmelev, A. A., J. F. Lynch, Y.-T. Lin, and H. Schmidt. 2014. Three-dimensional coupled mode analysis of internal-wave acoustic ducts. J. Acoust. Soc. Am., 135, 2497-2512. doi: 10.1121/1.4869847 
Skarsoulis, E. K., B. D. Cornuelle, and M. A. Dzieciuch. 2009. Travel-time sensitivity kernels in long-range propagation. J. Acoust. Soc. Am., 126, 2223-2233. doi: 10.1121/1.3224835

Spiesberger, J. L., R. C. Spindel and K. Metzger. 1980. Stability and identification of ocean acoustic multipaths. J. Acoust. Soc. Am., 67, 2011-2017. doi: 10.1121/1.384441

Udovydchenkov, I. A., T. F. Duda, S. C. Doney and I. D. Lima. 2010. Modeling deep ocean shipping noise in varying acidity conditions. J. Acoust. Soc. Am., 128, EL130-136. doi: 10.1121/1.3402284

White, A. W., R. K. Andrew, J. A. Mercer, P. F. Worcester, M. A. Dzieciuch, and J. A. Colosi. 2013. Wavefront intensity statistics for $284-\mathrm{Hz}$ broadband transmissions to 107-km range in the Philippine Sea: observations and modeling. J. Acoust. Soc. Am., 134, 3347-3358. doi: 10.1121/1.4818886

$\mathrm{Xu}, \mathrm{G}$., and D. Di Iorio. 2011. The relative effects of particles and turbulence on acoustic scattering from deep-sea hydrothermal vent plumes. J. Acoust. Soc. Am., 130, 1856-1867. doi: 10.1121/ 1.3624816

Yamamoto, T. 1983. Acoustic propagation in the ocean with a poro-elastic bottom. J. Acoust. Soc. Am., 73, 1587-1596. doi: 10.1121/1.389422

Yevick, D., and D. J. Thomson. 1997. A hybrid split-step/finite-difference PE algorithm for variabledensity media. J. Acoust. Soc. Am., 101, 1328-1335. doi: 10.1121/1.418160

Zhou, J. X., X. Z. Zhang, and P. H. Rogers. 1991. Resonant interaction of sound wave with internal solitons in the coastal zone. J. Acoust. Soc. Am., 90, 2042-2054. doi: 10.1121/1.401632

Received: 14 December 2015; revised: 16 August 2016.

Editor's note: Contributions to The Sea: The Science of Ocean Prediction are being published separately in special issues of Journal of Marine Research and will be made available in a forthcoming supplement as Volume 17 of the series. 